\title{
MAXIMAL OPERATORS AND B.M.O. FOR BANACH LATTICES*
}

\author{
by J. GARCÍA-CUERVA, R. A. MACÍAS and J. L. TORREA
}

(Received 19th December 1996)

\begin{abstract}
We investigate the behaviour of the classical (non-smooth) Hardy-Littlewood maximal operator in the context of Banach lattices. We are mainly concerned with end-point results for $p=\infty$. Naturally, the main role is played by the space $B M O$. We analyze the range of the maximal operator in $B M O_{\mathbf{x}}$. This turns out to depend strongly on the convexity of the Banach lattice $X$. We apply these results to study the behaviour of the commutators associated to the maximal operator. We also consider the parallel results for the maximal fractional integral operator.
\end{abstract}

1991 Mathematics subject classification: 42A28, 46B20, 43A25.

\section{Introduction}

In our paper [8] we have introduced the Hardy-Littlewood property (sometimes abbreviated as H.L. property) of a Banach lattice and have characterized it by several boundedness properties of the lattice version $\mathcal{M}$ of the Hardy-Littlewood maximal operator or its smooth variants. We refer the reader to this paper and also to [12] for the basic notation and terminology concerning Banach lattices and function spaces.

As we explain in [8], the Hardy-Littlewood property is intimately connected to the U.M.D. property, which plays a central role in the development of Vector-Valued Fourier Analysis and, in spite of having been extensively studied (see [6] and [3]), still has some aspects that need to be completely understood. By studying systematically the Hardy-Littlewood property, we hoped to cast some light on the behaviour of Banach lattices regarding the U.M.D. property.

The key idea in [8] is that the maximal operator $\mathcal{M}$ has a smooth version, obtained by replacing the averages over balls by the convolutions with an appropriate approximate identity. This smooth version can be viewed as a vector-valued singular integral, and its boundedness properties are a consequence of the general theory which was originally set up in [1] and then fully developed in [14]. This latter paper will also be a basic reference for the present work. In particular, the notation for all our function spaces will be taken from it.

When one is interested in estimates on the Bochner-Lebesgue spaces $L_{\mathbf{x}}^{p}\left(\mathbb{R}^{n}\right)$, only the size matters, and it is irrelevant whether we look at $\mathcal{M}$ or to its smooth version

\footnotetext{
- The first and third authors were supported by DGICYT, Spain, under Grant PB94-149. The second author was supported by Ministerio de Educación, Spain, under Sabattical Grant SB-.
} 
which, for a positive function, is almost everywhere equivalent to $\mathcal{M}$ in size. However, when we deal with the endpoints $p=1$ or $p=\infty$, we know from the classical theory of singular integrals that the natural spaces should be $H_{\mathbf{x}}^{\mathrm{l}}\left(\mathbb{R}^{n}\right)$ or $B M O_{\mathbf{x}}\left(\mathbb{R}^{n}\right)$. Now in these spaces, smoothness is a very important ingredient. Actually, already in the scalar case, we know that the non-smooth operator is not bounded from $H^{1}$ to $L^{1}$, while the smooth operator is bounded (see [16]). This is the reason why the $H^{1} \rightarrow L^{1}$ and $L^{\infty} \rightarrow B M O$ estimates of $[8]$ are considered only for the smooth version.

In the present paper we analyze completely the behaviour of the non-smooth Hardy-Littlewood maximal operator $\mathcal{M}$ at $p=\infty$. This is done in Section 2, after the discussion of some general facts about the Hardy-Littlewood property, carried out in Section 1. One of the main results we obtain is that the boundedness of $\mathcal{M}$ from $L_{\mathrm{x}}^{\infty}$ to $B M O_{\mathrm{x}}$ and its boundedness in $B M O_{\mathrm{x}}$, are equivalent, and actually, each of them is equivalent to the Hardy-Littlewood property. These results are beyond the classical theory of vector-valued singular integrals and require, as an essential ingredient, the $p$-convexity of the lattice for some $p>1$. This $p$-convexity follows from the HardyLittlewood property, as we already proved in [8, Theorem 2.8]. Actually our methods, which are rather natural in the vector-valued setting, give a very direct and simple proof of the $B M O$ boundedness of the scalar Hardy-Littlewood operator, which was proved by Bennet, De Vore and Sharpley (see [2]).

Section 2 also contains a brief discussion of the fractional maximal operator $\mathcal{M}^{x}, 0<\alpha<n$. Since this operator is bounded by the corresponding fractional integral operator $I_{\alpha}$ and the latter is just the convolution with a positive, locally integrable kernel, all the size estimates in Lebesgue spaces other than $L^{\infty}$ are simply consequences of the corresponding estimates for the fractional integral and those are the same as in the scalar case. However, the situation becomes different at the endpoints. For example, $p$-convexity with $p \geq n / \alpha$ is required for $\mathcal{M}^{\alpha} f$ to be in $L_{\mathbf{x}}^{\infty}\left(\mathbb{R}^{n}\right)$ for every $f \in L_{\mathbf{x}}^{n / \alpha}\left(\mathbb{R}^{n}\right)$.

In Section 3, we present an example in the same spirit as the one given by Bourgain in connection with the U.M.D. condition (see [3]), which shows that, for a general lattice to have the H.L. property, $p$-convexity even with a high $p$ is not enough.

Still, one of the main motivations of the present paper is to point out the different behaviour at $p=\infty$ between $\mathcal{M}$ and a typical singular integral. If the main point of [8] was the fact that from the vector-valued point of view, $\mathcal{M}$ is nothing more than a singular integral, at a deeper level there are differences in behaviour between $\mathcal{M}$ and, say, the Hilbert transform. These differences are linked to the convexity of $\mathbb{X}$. If there is nothing better than 1-convexity, both operators behave in the same way. In the other extreme, which is when we have $\infty$-convexity as in the scalar case, the two operators are radically different. In the intermediate cases, there is a continuous scale that bridges the gap between the two types of behaviour. This is the same phenomenon that was present in [10] for a particular problem in the theory of the weights associated to these operators. Here we look at the image of $L_{\mathrm{x}}^{\infty}$ in $B M O_{\mathrm{x}}$ for these operators and see a difference in behaviour of the same nature than that in [10], whose connection with convexity becomes now evident. All this is discussed in Section 4, where we also give some applications to the study of the commutators associated to the non-smooth maximal operator. 


\section{The Hardy-Littlewood property}

Definition 1.1. Let $\mathbb{X}$ be a Banach lattice and let $J$ be a finite subset of the set $\mathbb{Q}_{+}$ of the positive rational numbers. Given a locally integrable function $f: \mathbb{R}^{n} \rightarrow \mathbb{X}$ (this means, of course, a strongly measurable $f$ such that the scalar function $y \rightarrow\|f(y)\|_{x}$ is locally integrable) we define:

$$
\mathcal{M}_{J} f(x)=\sup _{r \in J} \frac{1}{|B(x, r)|} \int_{B(x, r)}|f(y)| d y
$$

where $|B(x, r)|=c_{n} r^{n}$ is the Lebesgue measure of the ball $B(x, r)$.

We shall always denote the Lebesgue measure of a set $E$ by $|E|$.

Notice that the sup in definition 1.1 is a sup in the lattice $X$. This accounts for the need to take just a finite collection of radii $J$. This difficulty will disappear when we deal with the most relevant examples, which will turn out to be order complete (see Remark 1.4 below).

Definition 1.2. We shall say that a Banach lattice $X$ satisfies the Hardy-Littlewood (H.L.) property if there exists some $p_{0}, 1<p_{0}<\infty$ such that the operators $\mathcal{M}_{s}$ are uniformly bounded in $L_{\mathbf{X}}^{p_{0}}\left(\mathbb{R}^{n}\right)$, that is, the inequality:

$$
\left\|\mathcal{M}_{J} f\right\|_{L_{\mathbf{x}}^{p_{0}}\left(\mathbf{R}^{n}\right)} \leq C\|f\|_{L_{\mathbf{x}}^{p_{0}}\left(\mathbf{R}^{n}\right)}
$$

holds with $C$ independent of $J$.

Two remarks are in order here. They are explained in detail in [8].

Remark 1.3. The H.L. property does not depend on the dimension $n$. We shall keep $n$ fixed with the understanding that its particular value is irrelevant. Also, the same notion is obtained if the torus $\mathbb{T}^{n}$ is used in place of $\mathbb{R}^{n}$.

Remark 1.4. Let $\left(\Omega, \sum, \mu\right)$ be a complete $\sigma$-finite measure space. If $\mathbb{X}$ is a Köthe function space of functions on $\Omega$ and $f: \mathbb{R}^{n} \rightarrow \mathbb{X}$ is a locally integrable function, it is clear that $\mathcal{M}_{f} f(x)$ is a function of $\omega$ given by:

$$
\mathcal{M}_{J} f(x)(\omega)=\sup _{r \in J} \frac{1}{|B(x, r)|} \int_{B(x, r)}|f(y, \omega)| d y
$$

where sup is now the sup in the order of $\mathbb{R}$. In this situation we can see $f$ and $\mathcal{M}_{J} f$ as scalar functions on $\mathbb{R}^{n} \times \Omega$.

Moreover, in this case we can define the operator $\mathcal{M}$ given by:

$$
\mathcal{M} f(x, \omega)=\sup _{r \in \mathbf{Q}_{+}} \frac{1}{|B(x, r)|} \int_{B(x, r)}|f(y, \omega)| d y
$$


We recall that a Köthe function space is said to have the Fatou property (see [12]) if everytime we have a sequence of functions $f_{n} \in \mathbb{X}$, such that $f_{n}(\omega) \geq 0$ for a.e. $\omega, f_{n}(\omega) \uparrow f(\omega)$ for a.e. $\omega$ and also $\sup _{n}\left\|f_{n}\right\|_{\mathbf{X}}<\infty$, then we have $f \in \mathbb{X}$ and $\|f\|_{\mathbf{X}}=\lim _{n}\left\|f_{n}\right\|_{\mathbf{X}}$.

It is a simple consequence of Lebesgue's monotone convergence theorem for scalar functions that the space $L_{\mathbf{X}}^{p_{0}}\left(\mathbb{R}^{n}\right)$ has the Fatou property provided $\mathbb{X}$ has it. Therefore, $a$ Köthe function space having the Fatou property satisfies the H.L. property if and only if there exists some $p_{0}, 1<p_{0}<\infty$ such that $\mathcal{M}$ is bounded in $L_{\mathbf{X}}^{p_{0}}\left(\mathbb{R}^{n}\right)$.

We shall need to consider as auxiliary operators, smooth versions of $\mathcal{M}_{J}$ and $\mathcal{M}$ which we define next:

Definition 1.6. Let $\varphi:[0, \infty[\rightarrow \mathbf{R}$ be a smooth function such that

$$
\chi_{[0,1]}(t) \leq \varphi(t) \leq \chi_{[0,2]}(t)
$$

for every $t \geq 0$.

Let $\mathbb{X}$ be a Banach lattice, $J$ a finite subset of $\mathbb{Q}_{+}$and $f: \mathbb{R}^{n} \rightarrow \mathbb{X}$ locally integrable. We define

$$
\mathcal{M}_{\varphi, J} f(x)=\sup _{r \in J}\left|\frac{1}{c_{0} r^{n}} \int_{\mathbf{R}^{n}} \varphi\left(\frac{|x-y|}{r}\right) f(y) d y\right|,
$$

$x \in \mathbb{R}^{n}$, where $c_{0}=\int_{\mathbb{R}^{n}} \varphi(|x|) d x$.

If $\mathbb{X}$ is a Köthe space of functions on $\Omega$, we can also define:

$$
\mathcal{M}_{\varphi} f(x, \omega)=\sup _{r \in \mathbf{Q}_{+}}\left|\frac{1}{c_{0} r^{n}} \int_{\mathbf{R}^{n}} \varphi\left(\frac{|x-y|}{r}\right) f(y, \omega) d y\right|
$$

In the definition of the $\mathcal{M}_{\varphi, J}$ 's, the sup and the $\|$ are those in $\mathbb{X}$, while in the definition of $\mathcal{M}_{\varphi}$ they are the corresponding ones in $\mathbb{R}$.

Remark 1.7. If in Definition 1.6 we allow $\varphi=\chi=\chi_{[0.1]}$, we obtain operators $\mathcal{M}_{\chi, J}$ and $\mathcal{M}_{\chi}$, which differ only from $\mathcal{M}_{J}$ or $\mathcal{M}$ in the fact that the bars of the absolute value are placed outside of the integrals rather than inside of them. This difference will turn out to be irrelevant for our results, so that many times, rather than working with $\mathcal{M}_{J}$ or $\mathcal{M}$ we shall be using $\mathcal{M}_{x, J}$ or $\mathcal{M}_{\chi}$ and we shall refer to them as the "nonsmooth") maximal operators "with the bars outside".

The main result of [8] was the following

Theorem 1.8. Given a Banach lattice $\mathbb{X}$ and a smooth function $\varphi$ as in the previous definition, the following conditions are equivalent: 
(I) $\mathbb{X}$ has the H.L. property.

(2) There exists some $p_{0}, 1<p_{0}<\infty$, such that

$$
\left\|\mathcal{M}_{\varphi, J} f\right\|_{L_{\mathbf{X}}^{p_{0}}\left(\mathbf{R}^{n}\right)} \leq C_{p_{0}}\|f\|_{L_{\mathbf{X}}^{p_{0}}\left(\mathbf{R}^{n}\right)} .
$$

(3) For every $p, 1<p<\infty$

$$
\left\|\mathcal{M}_{J} f\right\|_{L_{\mathbf{x}}^{p}\left(\mathbf{R}^{n}\right)} \leq C_{p}\|f\|_{L_{\mathbf{x}}^{p}\left(\mathbf{R}^{n}\right)} .
$$

(4) For every $p, 1<p<\infty$

$$
\left\|\mathcal{M}_{\varphi, J} f\right\|_{L_{\mathbf{X}}^{p}\left(\mathbf{R}^{n}\right)} \leq C_{p}\|f\|_{L_{\mathbf{X}}^{p}\left(\mathbf{R}^{n}\right)} .
$$

(5) $\left|\left\{x \in \mathbb{R}^{n}:\left\|\mathcal{M}_{J} f(x)\right\|_{\mathbf{X}}>\lambda\right\}\right| \leq \frac{c}{\lambda} \int_{\mathbf{R}^{n}}\|f(x)\|_{\mathbf{X}} d x$.

(6) $\left|\left\{x \in \mathbb{R}^{n}:\left\|\mathcal{M}_{\varphi, J} f(x)\right\|_{X}>\lambda\right\}\right| \leq \frac{c}{\lambda} \int_{\mathbb{R}^{n}}\|f(x)\|_{\mathbf{X}} d x$.

(7) $\left\|\mathcal{M}_{\varphi, J} f\right\|_{L_{\mathbf{x}}^{1}\left(\mathbf{R}^{n}\right)} \leq C\|f\|_{H_{\mathbf{x}}^{1}\left(\mathbf{R}^{n}\right)}$.

(8) $\left\|\mathcal{M}_{\varphi, J} f\right\|_{B M O_{\mathbf{x}}\left(\mathbf{R}^{n}\right)} \leq C\|f\|_{L_{\mathbf{x}}^{\infty}\left(\mathbf{R}^{n}\right)}$.

(9) If $w$ is an $A_{\infty}$ weight in $\mathbb{R}^{n}$ and $0<p<\infty$,

$$
\int_{\mathbf{R}^{n}}\left\|\mathcal{M}_{\varphi, J} f(x)\right\|_{\mathbf{X}}^{p} w(x) d x \leq C_{p}(w) \int_{\mathbf{R}^{n}}\left(M\left(\|f\|_{\mathbf{X}}\right)(x)\right)^{p} w(x) d x,
$$

where $M$ is the Hardy-Littlewood maximal operator in $\mathbb{R}^{n}$, which is applied to the scalar function $x \mapsto\|f(x)\|_{\mathbf{x}}$.

(10) For every cube $Q$ (with sides parallel to the coordinate axes, as we shall always assume) and every function $f \in L_{\mathbf{x}}^{\infty}\left(\mathbb{R}^{n}\right)$ having support contained in $Q$, we have:

$$
\int_{Q}\left\|\mathcal{M}_{\varphi, J} f(x)\right\|_{\mathbf{X}} d x \leq C\|f\|_{L_{\mathbf{x}}^{\infty}}|Q|
$$

The constants $C, C_{p}, C_{p}(w)$ (not the same at each occurrence) depend on $\mathbb{X}, \varphi, p$ or $w$, but do not depend on $J$.

Proof. The detailed proof is given in [8]. Here we shall only give the main features and we shall introduce some tools to be used in the rest of the paper. For $\mathbb{X}$-valued functions $f$, which satisfy $f(x) \geq 0$ a.e., we have

$$
\mathcal{M}_{J} f(x) \leq C \mathcal{M}_{\varphi, J} f(x) \leq C \mathcal{M}_{2 J} f(x), \quad x \in \mathbb{R}^{n}
$$

These inequalities immediately yield (1) $\Leftrightarrow(2),(3) \Leftrightarrow(4)$ and (5) $\Leftrightarrow(6)$.

Next we shall prove that $(2) \Rightarrow(6)$.

We just need to consider one fixed $J$ and see that the boundedness of $\mathcal{M}_{\varphi, J}$ in $L_{\mathbf{X}}^{p_{0}}$ implies that it is bounded from $L_{\mathbf{X}}^{1}$ to weak- $L_{\mathbf{X}}^{1}$ with a constant depending only on its norm as an operator bounded in $L_{\mathbf{X}}^{p^{O}}$.

In order to do that, we shall consider an operator $T_{J}$ sending $\mathbb{X}$-valued functions into functions taking values in the Banach space $X(J)$, consisting of the sequences $\left(x_{r}\right)_{r \in J}$ of elements $x_{r} \in \mathrm{X}$ with $\left\|\left(x_{r}\right)_{r \in J}\right\|_{\mathrm{X}(J)}=\left\|\sup _{r}\left|x_{r}\right|\right\|_{\mathrm{X}}$. 
For $f: \mathbb{R}^{n} \rightarrow \mathbb{X}$ locally integrable, we define:

$$
T_{J} f(x)=\left(\frac{1}{c_{0} r^{n}} \int_{\mathrm{R}^{n}} \varphi\left(\frac{|x-y|}{r}\right) f(y) d y\right)_{r \in J}
$$

This is to be viewed as a linearization of the operator $\mathcal{M}_{\varphi, J}$. Since

$$
\left\|T_{J} f(x)\right\|_{\mathbf{X}(J)}=\left\|\mathcal{M}_{\varphi, J} f(x)\right\|_{\mathbf{X}}
$$

the boundedness of $\mathcal{M}_{\varphi, J}$ in $L_{\mathbf{X}}^{p_{0}}\left(\mathbb{R}^{n}\right)$ is equivalent to the boundedness of $T_{J}$ from $L_{\mathbf{X}}^{p_{0}}\left(\mathbb{R}^{n}\right)$ to $L_{\mathbf{X}(J)}^{p_{0}}\left(\mathbb{R}^{n}\right)$.

But $T_{J}$ is a linear operator given by convolution with a kernel $K_{J}(x) \in \mathcal{L}(\mathbb{X}, \mathbb{X}(J))$ (bounded linear operators from $\mathbb{X}$ to $\mathbb{X}(J)$ ), namely:

$$
\begin{gathered}
K_{J}(x) v=\left(\frac{1}{c_{0} r^{n}} \varphi\left(\frac{|x|}{r}\right) v\right)_{r \in J} ; \quad v \in \mathbb{X} \\
\left\|K_{J}(x)\right\|_{\mathcal{L} \mathbf{X}, \mathbf{X}(J))}=\sup _{r \in J} \frac{1}{c_{0} r^{n}} \varphi\left(\frac{|x|}{r}\right) .
\end{gathered}
$$

The smoothness of $\varphi$ guarantees that $K_{J}$ satisfies the so-called standard estimates for Calderón-Zygmund kernels, that is:

$$
\left\|K_{J}(x)\right\| \leq C|x|^{-n}
$$

and

$$
\left\|K_{J}(x)-K_{J}\left(x^{\prime}\right)\right\| \leq C\left|x-x^{\prime} \| x\right|^{-n-1} \text { for } 2\left|x-x^{\prime}\right|<|x|
$$

Note that these estimates are uniform in $J$, i.e., the constant $C$ does not depend on $J$. Now the theory of vector-valued Calderón-Zygmund operators, as given in [14] can be applied to $T_{J}$ to obtain

$$
\left|\left\{x \in \mathbb{R}^{n}:\left\|T_{J} f(x)\right\|_{\mathbf{X}(J)}>\lambda\right\}\right| \leq \frac{C}{\lambda} \int_{\mathbf{R}^{n}}\|f(x)\|_{\mathbf{X}} d x .
$$

But this is precisely (6).

That $(6) \Rightarrow(9)$ is obtained by getting, via Cotlar's inequality, a good- $\lambda$ inequality between the "singular integral" and the "maximal function" (see [9, Chapters 2 and 4]). Then, trivially (9) $\Rightarrow(4)$ and (4) $\Rightarrow(2)$. That way we have that (1), (2), (3), (4), (5), (6) and (9) are equivalent. Then (10) follows from (2) in a rather straightforward way.

Let us briefly recall how to derive (8) from (10), since this is the main type of estimate we shall be looking for in the rest of the paper.

Let $f \in L_{X}^{\infty}$. Given a cube $Q$ with centre $x_{0}$, we consider the cube $\tilde{Q}$ with the same 
centre and sidelength three times the diameter of $Q$, and decompose $f=f_{1}+f_{2}$ where $f_{1}=f \chi_{\grave{Q}}$. Then we have

$$
\begin{aligned}
& \frac{1}{|Q|} \int_{Q}\left\|\mathcal{M}_{\varphi, J} f(x)-\mathcal{M}_{\varphi, J} f_{2}\left(x_{0}\right)\right\|_{\mathbf{x}} d x \leq \frac{1}{|Q|} \int_{Q}\left\|T_{J} f(x)-T_{J} f_{2}\left(x_{0}\right)\right\|_{\mathbf{X}(J)} d x \\
& \quad \leq \frac{1}{|Q|} \int_{Q}\left\|T_{J} f_{1}(x)\right\|_{\mathbf{X}(J)} d x+\frac{1}{|Q|} \int_{Q}\left\|T_{J} f_{2}(x)-T_{J} f_{2}\left(x_{0}\right)\right\|_{\mathbf{X}(J)} d x
\end{aligned}
$$

The first term in this sum is handled by using (10) and the second term by using the standard estimates for $K_{J}$. That way we obtain (8).

Let us also recall briefly how to obtain (7) from (8), since this argument will also play a role in the sequel. We take an atom $a \in H_{X}^{1}$ and show that:

$$
\left\|\mathcal{M}_{\varphi, J} a\right\|_{L_{\mathbf{x}}^{1}\left(\mathbf{R}^{n}\right)}=\left\|T_{J} a\right\|_{L_{\mathbf{x}(J)}^{1}\left(\mathbf{R}^{n}\right)} \leq C
$$

That $a$ is an atom means that $a: \mathbb{R}^{n} \rightarrow \mathbb{X}$ is supported in a cube $Q$, with centre $x_{0}$, say; $a$ has also average 0 and it satisfies $\|a(x)\|_{\mathbf{x}} \leq 1 /|Q|$ a.e. For $x \notin \tilde{Q}$, we have:

$$
T_{J} a(x)=\int_{Q} K_{J}(x-y) a(y) d y=\int_{Q}\left(K_{J}(x-y)-K_{J}\left(x-x_{0}\right)\right) a(y) d y,
$$

which, together with the standard estimates gives:

$$
\left\|\mathcal{M}_{\varphi, J} a(x)\right\|_{\mathbf{x}} \leq C|Q|^{1 / n}\left|x-x_{0}\right|^{-n-1}
$$

and, consequently

$$
\int_{\mathbf{R}^{n} \backslash \tilde{Q}}\left\|\mathcal{M}_{\varphi, J} a(x)\right\|_{\mathbf{x}} d x \leq C
$$

On $\tilde{Q}$ we can use (8) which tells us that

$$
\left\|\mathcal{M}_{\varphi, J} a\right\|_{B M O_{\mathrm{x}}} \leq C|Q|^{-1}
$$

In particular

$$
\frac{1}{|\tilde{Q}|} \int_{\tilde{Q}}\left\|\mathcal{M}_{\varphi, J} a(x)-\left(\mathcal{M}_{\varphi, J} a\right)_{\tilde{Q}}\right\|_{\mathbf{x}} d x \leq C|Q|^{-1}
$$

But note also that if $Q^{*}$ is a cube adjacent to $\bar{Q}$ and with the same size

$$
\left\|\left(\mathcal{M}_{\varphi, J} a\right)_{\dot{Q}}-\left(\mathcal{M}_{\varphi, J} a\right)_{Q^{*}}\right\|_{\mathbf{x}} \leq C|Q|^{-1}
$$


as one sees by adding and subtracting the average on the smallest cube containing both $\tilde{Q}$ and $Q^{*}$.

Also, since $Q^{*} \subset \mathbb{R}^{n} \backslash \tilde{Q}$,

$$
\left\|\left(\mathcal{M}_{\varphi, J} a\right)_{Q^{*}}\right\|_{\mathbf{X}} \leq C|Q|^{-1}
$$

so that, actually

$$
\left\|\left(\mathcal{M}_{\varphi, J} a\right)_{\bar{Q}}\right\|_{\mathbf{x}} \leq C|Q|^{-1}
$$

and

$$
\frac{1}{|\tilde{Q}|} \int_{\tilde{Q}}\left\|\mathcal{M}_{\varphi, J} a(x)\right\|_{\mathrm{x}} d x \leq C|Q|^{-1}
$$

We get finally

$$
\int_{\mathbf{R}^{n}}\left\|\mathcal{M}_{\varphi, J} a(x)\right\|_{\mathbf{X}} d x=\int_{\bar{Q}}+\int_{\mathbf{R}^{n} \backslash \bar{Q}} \leq C .
$$

That (7) implies (10) is almost immediate. We just need to pass from a general bounded function to an atom by subtracting its average.

Thus (7), (8) and (10) are equivalent. They imply (2) by interpolation.

A large class of examples of H.L. Banach lattices is provided by those Köthe function spaces satisfying the condition known as U.M.D. (from "unconditional martingale differences"). Recall that a Banach space $\mathbb{X}$ is said to be U.M.D. if it satisfies an inequality

$$
\left\|\sum_{k=1}^{n} \varepsilon_{k} d_{k}\right\|_{L_{\mathrm{x}}^{p}} \leq C_{p, X}\left\|\sum_{k=1}^{n} d_{k}\right\|_{L_{\mathbf{x}}^{p}}
$$

for all $n \in \mathbb{N}, \varepsilon_{k}= \pm 1$ and for all $\mathbb{X}$-valued martingale differences $\left\{d_{k}\right\}_{k \geq 1}$, where $p$ is some exponent such that $1<p<\infty$ (see [6]).

The concrete relationship between the U.M.D. and H.L. properties for a Köthe function space is given in the following theorem due to J. Bourgain and J. L. Rubio de Francia (see [4 and 13]).

Proposition 1.9. For a Köthe function space $\mathbb{X}$ with the Fatou property, the following conditions are equivalent:

(a) $\mathbb{X}$ is U.M.D.

(b) Both $\mathbb{X}$ and its function space dual $\mathbb{X}^{\prime}$ satisfy the H.L. property. For a general Köthe function space, still (a) implies $(b)$.

We know that $\ell^{p}$ is U.M.D. provided $1<p<\infty$. Thus, for this range, $\ell^{p}$ is H.L. 
This fact follows from the inequality

$$
\left\|\left(\sum_{j=1}^{\infty}\left|M f_{j}\right|^{p}\right)^{1 / p}\right\|_{L^{q}\left(\mathbf{R}^{n}\right)} \leq C_{p . q}\left\|\left(\sum_{j=1}^{\infty}\left|f_{j}\right|^{p}\right)^{1 / p}\right\|_{L^{q}\left(\mathbf{R}^{n}\right)}
$$

due to C. Fefferman and E. M. Stein (see [7]). The simplest way to prove (1.10) for $q<p$ is to use the obvious result for $p=q$ plus the observation that we have a version of (1.10) for $p=\infty$, namely

$$
\left\|\sup _{j} M f_{j}\right\|_{L^{q}\left(\mathbf{R}^{n}\right)} \leq C_{p, \infty}\left\|\sup _{j}\left|f_{j}\right|\right\|_{L^{q}\left(\mathbb{R}^{n}\right)}
$$

Since $M$ is linearizable, we can interpolate (see [9, Chapter V, Section 1]), obtaining the result for $q<p$. Now Theorem 1.9 gives (1.10) for every $q, 1<q<\infty$, once we have it for one $q$. (1.11) tells us that $\ell^{\infty}$, and also $c_{0}$, have the H.L. property.

The main example of a Banach lattice without the H.L. property is given by

Proposition 1.12. $\ell^{1}$ does not have the H.L. property.

The proof is in [8, Proposition 2.4], where it is used to derive the convexity result that we shall explain next, which will turn out to be basic in what follows.

Definition 1.13. A Banach lattice $\mathbb{X}$ is said to be $p$-convex $1 \leq p<\infty$, if the following inequality holds:

$$
\left\|\left(\sum_{j=1}^{m}\left|x_{j}\right|^{p}\right)^{1 / p}\right\|_{\mathbf{x}} \leq C_{p}\left(\sum_{j=1}^{m}\left\|x_{j}\right\|_{\mathbf{x}}^{p}\right)^{1 / p}
$$

with a constant $C_{p}$ independent of $m$.

It is said to be $\infty$-convex if we have

$$
\left\|\sup _{1 \leq j \leq m}\left|x_{j}\right|\right\|_{\mathrm{X}} \leq C_{\infty} \sup _{1 \leq j \leq m}\left\|x_{j}\right\|_{\mathrm{X}}
$$

When $\mathbb{X}$ is a lattice of functions or, more generally, when $\mathbb{X}$ is order continuous, the concrete representation of the lattice allows us to define $\left(\sum_{j=1}^{m}\left|x_{j}\right|^{p}\right)^{1 / p}$ in the obvious way. However, for a general lattice, these expressions need to be defined (see $[12,1 . d])$.

In Definition 1.13, the sums can be replaced by integrals and it is precisely in this way that we shall be using the $p$-convexity.

Here is the basic convexity result (Theorem 2.8 in [8]). 
Theorem 1.14. If a Banach lattice $\mathbb{X}$ has the H.L. property, then $\mathbb{X}$ is p-convex for some $p>1$.

Remark 1.15. In general, $\mathcal{M}$ is not bounded in $L_{\mathbf{x}}^{\infty}$. In [8] we give an example which actually proves that if $\mathrm{X}=\ell^{p}, \mathcal{M}$ is bounded in $L_{\mathrm{x}}^{\infty}$ only when $p=\infty$.

We finish this section by proving a stability result for the H.L. property for Köthe function spaces.

Definition 1.16. Given a Köthe space $\mathbb{X}$ of measurable functions on a measure space $\Omega$, and given $s>0$, we define $\mathbb{X}^{3}$ to be the lattice consisting of those measurable functions $f$ such that $|f|^{1 / s} \in \mathbb{X}$. We also define

$$
\|f\|_{\mathbf{X}^{s}}=\left\||f|^{1 / s}\right\|_{\mathbf{X}}^{s} .
$$

Proposition 1.17. Let $s>1$. Then $\mathbb{X}^{s}$ is a Banach lattice if and only if $\mathbb{X}$ is s-convex.

The proof is straightforward.

J. L. Rubio de Francia proved (see [13]) that if $\mathbb{X}$ is U.M.D., then there is some $\varepsilon>0$, such that $\mathbb{X}^{s}$ is also U.M.D. for every $s$ such that $1<s<1+\varepsilon$. Now we can see that the H.L. property is also a Rubio de Francia property in the sense that the following result holds.

Theorem 1.18. Let $\mathbb{X}$ be a Köthe space with the Fatou property. Suppose that $\mathbb{X}$ satisfies the H.L. property. Then there is some $\varepsilon>0$, such that $\mathbb{X}^{s}$ also satisfies the H.L. property for every $s$ in the range $1<s<1+\varepsilon$.

Proof. First of all we observe that it is enough to prove the following.

Claim. The operator $\mathcal{M}_{s}$ defined by $\mathcal{M}_{s}(f)=\mathcal{M}\left(|f|^{s}\right)^{1 / s}$ is bounded in $L_{\mathbf{x}}^{p}$ for some $s>1$ and every $p$ such that $s<p<\infty$.

Indeed, assuming that the claim is true, we can write, for $q>1$ and our $s$

$$
\begin{aligned}
\int_{\mathbf{R}^{n}}\|\mathcal{M}(f)(x)\|_{\mathbf{X}^{s}}^{q} d x & =\int_{\mathbf{R}^{n}}\left\||\mathcal{M}(f)(x)|^{1 / s}\right\|_{\mathbf{X}}^{s q} d x=\left(\text { setting } g=|f|^{1 / s}\right) \\
& =\int_{\mathbf{R}^{n}}\left\|\mathcal{M}_{s}(g)(x)\right\|_{\mathbf{X}}^{s q} d x \leq C \int_{\mathbf{R}^{n}}\|g(x)\|_{\mathbf{X}}^{s q} d x=C \int_{\mathbf{R}^{n}}\|f(x)\|_{\mathbf{X}^{s}}^{q} d x .
\end{aligned}
$$

Thus we get that $X^{s}$ satisfies the H.L. property.

Now, in order to prove the claim, we start from the boundedness of $\mathcal{M}$ in $L_{\mathrm{X}}^{p}$ and use the Rubio de Francia algorithm as in [9, Chapter IV] to construct 


$$
F(x, \omega)=\sum_{j=0}^{\infty} \frac{\mathcal{M}^{j} f(x, \omega)}{(2\|\mathcal{M}\|)^{j}} \geq f(x, \omega)
$$

which also satisfies

$$
\|F\|_{L_{\mathrm{x}}^{p}} \leq 2\|f\|_{L_{\mathrm{x}}^{p}} \text { and } \quad \mathcal{M} F(x, \omega) \leq 2\|\mathcal{M}\| F(x, \omega) \quad \text { for a.e. }(x, \omega) \in \mathbb{R}^{n} \times \Omega
$$

This tells us that $x \mapsto F(x, \omega)$ is an $A_{1}$ weight uniformly in $\omega \in \Omega$. Consequently, all these weights satisfy uniform reverse Hölder's inequalities (see [9, Chapter IV]). By taking $s>1$ for which these reverse Hölder's inequalities hold, we get

$$
\mathcal{M}_{s}(f)(x, \omega) \leq \mathcal{M}_{s}(F)(x, \omega) \leq C \mathcal{M} F(x, \omega) .
$$

Thus

$$
\int_{\mathbf{R}^{n}}\left\|\mathcal{M}_{s}(f)(x)\right\|_{\mathbf{X}}^{p} d x \leq C \int_{\mathbf{R}^{n}}\|\mathcal{M}(F)(x)\|_{\mathbf{X}}^{p} d x \leq C\|F\|_{L_{\mathbf{X}}^{p}} \leq C\|f\|_{L_{\mathbf{x}}^{p}},
$$

which establishes the claim and completes the proof of the theorem.

\section{The classical (non-smooth) maximal operator}

In this section we present the new results we have obtained for the non-smooth Hardy-Littlewood maximal operator, which fall outside of the Calderón-Zygmund theory.

Theorem 2.1. If $\mathbb{X}$ is a Banach lattice with the H.L. property, then the operators $\mathcal{M}$, are uniformly bounded from $L_{\mathbf{X}}^{\infty}\left(\mathbb{R}^{n}\right)$ to $B M O_{\mathbf{X}}\left(\mathbb{R}^{n}\right)$.

Proof. Since $\mathcal{M}_{J}(f)=\mathcal{M}_{x, J}(|f|)$, we can restrict our attention to $f \geq 0$ and use the linearized operators $T_{J}$ corresponding to $\mathcal{M}_{\chi, J}$. So, let $f \in L_{\mathbf{X}}^{\infty}\left(\mathbb{R}^{n}\right), f \geq 0$. Given $Q$, we write, exactly as in the proof that $(10) \Rightarrow(8)$ in Theorem $1.8, f=f_{1}+f_{2}$, where $f_{1}=f \chi_{\bar{Q}}$. Let $x_{0}$ be the centre of $Q$ and $2 \delta$ its diameter. Then

$$
\begin{aligned}
\frac{1}{|Q|} \int_{Q}\left\|\mathcal{M}_{J} f(x)-\mathcal{M}_{J} f_{2}\left(x_{0}\right)\right\|_{\mathbf{X}} d x & \leq \frac{1}{|Q|} \int_{Q}\left\|T_{J} f(x)-T_{J} f_{2}\left(x_{0}\right)\right\|_{\mathbf{x}(J)} d x \\
& \leq \frac{1}{|Q|} \int_{Q}\left\|T_{J} f_{1}(x)\right\|_{\mathbf{x}(J)} d x+\frac{1}{|Q|} \int_{Q}\left\|T_{J} f_{2}(x)-T_{J} f_{2}\left(x_{0}\right)\right\|_{\mathbf{x}(J)} d x
\end{aligned}
$$

By using Hölder's inequality with any $p>1$ and applying the H.L. property of $\mathrm{X}$, we can estimate the first term in this sum by 


$$
\left(\frac{1}{|Q|} \int_{Q}\left\|\mathcal{M}_{J} f_{1}(x)\right\|_{\mathrm{x}}^{p} d x\right)^{1 / P} \leq C\left(\frac{1}{|Q|} \int_{\bar{Q}}\|f(x)\|_{\mathbf{x}}^{p} d x\right)^{1 / p} \leq C\|f\|_{L_{\mathbf{x}}^{\infty}}
$$

The second term is bounded by the average over $Q$ of the $\mathbb{X}$-norm of

$$
\sup _{r \in J} \frac{C}{r^{n}} \int_{\mathbf{R}^{n}}\left|\chi\left(\frac{|x-y|}{r}\right)-\chi\left(\frac{\left|x_{0}-y\right|}{r}\right)\right|\left|f_{2}(y)\right| d y
$$

where $x \in Q$ and $y \in \mathbb{R}^{n} \backslash \tilde{Q}$. The integral will vanish unless, either $|x-y|<r$ and $\left|x_{0}-y\right|>r$, or else $|x-y|>r$ and $\left|x_{0}-y\right|<r$. In the first case $r<\left|x_{0}-y\right| \leq|x-y|+$ $\left|x-x_{0}\right|<r+\delta$, and in the second $r>\left|x_{0}-y\right| \geq|x-y|-\left|x_{0}-x\right|>r-\delta$. Therefore, the integral is actually restricted to $r-\delta<\left|x_{0}-y\right|<r+\delta$. On the other hand, this region must intersect the support of $f_{2}$. So, we must have $\left|x_{0}-y\right|>3 \delta$, for some $y$ in the region. It follows that $r+\delta>3 \delta$. In other words, only those $r>2 \delta$ are involved. Consequently, (2.2) is bounded by

$$
\begin{aligned}
\sup _{r>2 \delta} \frac{C}{r^{n}} \int_{r-\delta<\left|x_{0}-y\right|<r+\delta} & |f(y)| d y \\
\leq & \sup _{r>2 \delta} \frac{C}{r^{n}}\left(\int_{r-\delta<\left|x_{0}-y\right|<r+\delta}|f(y)|^{s} d y\right)^{1 / s}\left\{(r+\delta)^{n}-(r-\delta)^{n}\right\}^{1 / s^{\prime}} \\
& =C \sup _{r>2 \delta}\left(\int_{\mathbf{R}^{n}} h_{r}\left(x_{0}-y\right)|f(y)|^{s} d y\right)^{1 / s},
\end{aligned}
$$

where

$$
h_{r}(x)=\frac{\left\{(r+\delta)^{n}-(r-\delta)^{n}\right\}^{s / s^{\prime}}}{r^{n s}} \chi_{r-\delta<|x|<r+\delta}(x) \leq \frac{C \delta^{s / s^{\prime}}}{|x|^{n+s / s^{\prime}}} \chi_{|x|>\delta}(x)=H(x) .
$$

But now $H$ is just an integrable kernel with $L^{1}$-norm independent of $\delta$. It follows that the $\mathbb{X}$-norm of (2.2) is bounded by

$$
\begin{aligned}
\left\|\left(\int_{\mathbf{R}^{n}} H\left(x_{0}-y\right)|f(y)|^{s} d y\right)^{1 / s}\right\|_{\mathbf{X}} & \leq(\text { if } \mathbb{X} \text { is s-convex }) \\
& \leq\left(\int_{\mathbf{R}^{n}} H\left(x_{0}-y\right)\|f(y)\|_{\mathbf{X}}^{s} d y\right)^{1 / s} \leq C\|f\|_{L_{\mathbf{X}}^{\infty}}
\end{aligned}
$$

Only a small modification is needed to obtain

Theorem 2.3. If $\mathbb{X}$ is a Banach lattice with the H.L. property, then the operators $\mathcal{M}_{\mathrm{J}}$ are uniformly bounded in $B M O_{\mathbf{x}}\left(\mathbb{R}^{n}\right)$.

Proof. Since $f \in B M O_{\mathbf{x}} \Rightarrow|f| \in B M O_{\mathbf{x}}$ and $\|\mid f\|_{B M O_{\mathbf{x}}} \leq\|f\|_{B M O_{\mathbf{x}}}$, we may still 
restrict our attention to $f \in B M O_{\mathbf{x}}\left(\mathbb{R}^{n}\right), f \geq 0$ and use the linearization $T_{J}$ of $\mathcal{M}_{x, J}$. Given a cube $Q$, with $x_{0}$ and $\delta$ having the same meaning as in the proof of Theorem 2.1, we decompose $f$ as follows

$$
f=f-f_{Q}+f_{Q}=\left(f-f_{Q}\right) \chi_{\bar{Q}}+\left(f-f_{Q}\right) \chi_{\mathbf{R}^{n} \backslash \bar{Q}}+f_{Q}=g_{1}+g_{2},
$$

where $f_{Q}$ denotes the average of $f$ over $Q$ and $g_{1}=\left(f-f_{Q}\right) \chi_{\bar{Q}}$. Let us also write $h_{2}=\left(f-f_{Q}\right) \chi_{\mathbf{R}^{n} \backslash \bar{Q}}$. Then

$$
\begin{array}{rl}
\frac{1}{|Q|} \int_{Q}\left\|\mathcal{M}_{J} f(x)-\mathcal{M}_{\chi, J} g_{2}\left(x_{0}\right)\right\|_{\mathbf{x}} & d x \leq \frac{1}{|Q|} \int_{Q}\left\|T_{J} f(x)-T_{J} g_{2}\left(x_{0}\right)\right\|_{\mathbf{X}(J)} d x \\
& \leq \frac{1}{|Q|} \int_{Q}\left\|T_{J} g_{1}(x)\right\|_{\mathbf{x}(J)} d x+\frac{1}{|Q|} \int_{Q}\left\|T_{J} g_{2}(x)-T_{J} g_{2}\left(x_{0}\right)\right\|_{\mathbf{X}(J)} d x
\end{array}
$$

As before, using the H.L. property, we estimate the first term in this sum by

$$
\left(\frac{1}{|Q|} \int_{Q}\left\|\mathcal{M}_{J} g_{1}(x)\right\|_{\mathbf{x}}^{p} d x\right)^{1 / p} \leq C\left(\frac{1}{|Q|} \int_{\bar{Q}}\left\|f(x)-f_{Q}\right\|_{\mathbf{x}}^{p} d x\right)^{1 / p} \leq C\|f\|_{B M O_{\mathbf{X}}}
$$

The second term equals $\frac{1}{|Q|} \int_{Q}\left\|T_{J} h_{2}(x)-T_{J} h_{2}\left(x_{0}\right)\right\|_{\mathbf{X}(J)} d x$, whose integrand we bound, just as in the proof of Theorem 2.1 by

$$
\begin{aligned}
\left(\int_{\mathbf{R}^{n} \backslash \bar{Q}} H\left(x_{0}-y\right)\left\|f(y)-f_{Q}\right\|_{\mathbf{X}}^{s} d x\right)^{1 / s} \leq & \left\{\sum_{k=2}^{\infty} \int_{2^{k+1} Q \backslash 2^{k} Q} H\left(x_{0}-y\right)\left\|f(x)-f_{Q}\right\|_{\mathbf{X}}^{s} d x\right\}^{1 / s} \\
\leq & \left\{\sum_{k=2}^{\infty}\left(\int_{2^{k+1} Q \backslash 2^{k} Q}\left|H\left(x_{0}-y\right)\right|^{t} d y\right)^{1 / t}\left|2^{k+1} Q\right|^{1 / t^{\prime}}\right\}^{1 / s} \\
& \times\left(\frac{1}{\left|2^{k+1} Q\right|} \int_{2^{k+1} Q}\left\|f(y)-f_{Q}\right\|_{\mathbf{X}}^{s t^{\prime}} d x\right)^{1 /\left(s^{\prime}\right)}
\end{aligned}
$$

where $t>1$, and $2^{j} Q$ denotes the cube concentric with $Q$ with diameter $2^{j}$ times that of $Q$, that is, $2^{j+1} \delta$. But

$$
\begin{aligned}
& \left(\frac{1}{\left|2^{k+1} Q\right|} \int_{2^{k+1} Q}\left\|f(y)-f_{Q}\right\|_{\mathbf{x}}^{s t^{\prime}} d x\right)^{1 /\left(s t^{\prime}\right)} \\
& \quad=\left(\frac{1}{\left|2^{k+1} Q\right|} \int_{2^{k+1} Q}\left\|f(y)-f_{2^{k+1} Q}+f_{2^{k+1} Q}-\cdots-f_{Q}\right\|_{\mathbf{x}}^{s t^{\prime}} d x\right)^{1 /\left(s s^{\prime}\right)} \leq C k\|f\| B M O_{\mathbf{x}}
\end{aligned}
$$

and 


$$
\left(\int_{2^{k+1} Q 2^{k} Q}\left|H\left(x_{0}-y\right)\right|^{t} d y\right)^{1 / t} \leq \frac{C \delta^{s / s}\left[\left(2^{k+1} \delta\right)^{n}-\left(2^{k} \delta\right)^{n}\right]^{1 / t}}{\left(2^{k} \delta\right)^{n+\frac{3}{3}}}=C 2^{k\left(\frac{\eta}{7}-n-\frac{3}{3}\right)} \delta^{n-n}
$$

Thus the second term is bounded by

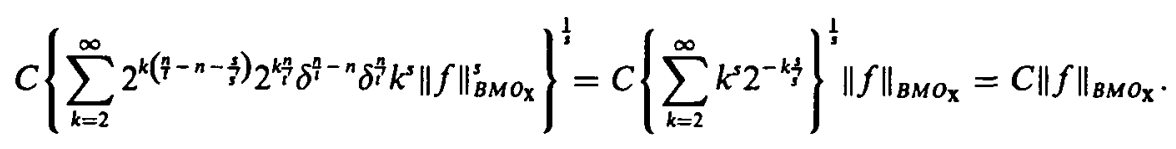

Theorem 2.3 is a vector-valued version of the theorem of Bennett, De Vore and Sharpley (see [2]), which becomes a particular case.

Actually, as we shall presently see, the converses of Theorems 2.1 and 2.3 are also true, so that they provide other characterizations of the H.L. property. We just need to prove the following.

Theorem 2.4. Let $\mathbb{X}$ be a Banach lattice for which the operators $\mathcal{M}_{J}$ are uniformly bounded from $L_{\mathbf{X}}^{\infty}\left(\mathbb{R}^{n}\right)$ to $B M O_{\mathbf{X}}\left(\mathbb{R}^{n}\right)$. Then $\mathbb{X}$ satisfies the H.L. property.

Proof. First of all we observe that our hypothesis implies that $\mathbb{X}$ is $p$-convex for some $p>1$. To prove this we proceed very much like in [8]. We start by seeing that $\mathcal{M}$ is not bounded from $L_{\ell^{\prime}}^{\infty}$ to $B M O_{\ell^{\prime}}$. The way to establish this fact, for $n=1$, as we may assume, is to examine more closely the counterexample given in [8, Proposition 2.4]. Let $m$ be a fixed natural number and consider the function $F_{m}: \mathbb{R} \rightarrow \ell^{1}$ given by:

$$
F_{m}(x)=\left(f_{1}(x), f_{2}(x), \ldots, f_{m}(x), 0,0, \ldots\right),
$$

where

$$
f_{j}(x)=\chi_{\left[\frac{[-1,4 \pi}{m}\right]}(x), \quad 1 \leq j \leq m
$$

Then

$$
\left\|F_{m}(x)\right\|_{\ell^{1}}=\sum_{j=1}^{m} f_{j}(x)=\chi_{[0,1]}(x)
$$

and

$$
\left\|F_{m}\right\|_{L_{\ell^{\prime}}^{\infty}(\mathbf{R})}=1 .
$$

On the other hand, for every $1 \leq j \leq m$,

$$
M f_{j}(x)= \begin{cases}1 & \text { if } x \in\left[\frac{j-1}{m}, \frac{i}{m}\right] \\ \frac{1}{j-m x} & \text { if } x \in\left[0, \frac{j-1}{m}\right] \\ \frac{1}{m x-j+1} & \text { if } x \in\left[\frac{1}{m}, 1\right]\end{cases}
$$


from which we get

$$
\int_{0}^{1} M f_{j}(x) d x=\frac{1}{m}(1+\log j+\log (m-j+1))=a_{j} .
$$

Now assume $m-j+1 \leq j$, and let $b_{j}<\frac{j-1}{m}$ be such that $M f_{j}\left(b_{j}\right)=a_{j}$, in other words $a_{j}=\left(j-m b_{j}\right)^{-1}$. Then

$$
\int_{0}^{j / m}\left|M f_{j}(x)-a_{j}\right| \geq \int_{b_{j}}^{\frac{j-1}{m}}+\int_{\frac{i-1}{m}}^{\frac{j}{m}}=\frac{1}{m} \log \left(\frac{1}{a_{j}}\right)
$$

A simple computation yields $a_{j} \leq m^{-1 / 2}$ for $m$ large. Therefore, if we carry this estimate to $(2.5)$, we obtain

$$
\int_{0}^{j / m}\left|M f_{j}(x)-a_{j}\right| \geq \frac{1}{2 m} \log m
$$

and, consequently, writing $I=[0,1]$ for simplicity

$$
\int_{l}\left\|\mathcal{M}\left(F_{m}\right)-\left(\mathcal{M}\left(F_{m}\right)\right)_{l}\right\|_{\ell^{1}} \geq \sum_{\frac{m+1}{2}<j \leq m} \int_{0}^{1}\left|M f_{i}-a_{j}\right| \geq \frac{1}{4} \log m
$$

which finishes the proof of the claim. Next, after we know that $\mathcal{M}$ is not bounded from $L_{\ell^{1}}^{\infty}$ to $B M O_{\ell^{1}}$, we prove that $\mathbb{X}$ is $p$-convex for some $p>1$ exactly as in [8, Theorem 2.8 ], using the fact that if this were not the case, $\mathbb{X}$ would contain 'almost isometric' copies of $\ell_{n}^{\prime}$ for every $n$.

Finally, in order to establish the H.L. property for $\mathbb{X}$, we are going to see that our $\mathbb{X}$ satisfies condition (10) in Theorem 1.8. Of course we just need to check it for $\mathcal{M}_{\boldsymbol{J}}$ in place of $\mathcal{M}_{\varphi, J}$. We take a cube $Q$ and a bounded $\mathbb{X}$-valued function having support in $Q$. We assume temporarily that the average of $f$ over $Q$ equals 0 and estimate, for $x \notin \tilde{Q}$,

$$
\begin{aligned}
\left\|\mathcal{M}_{x^{\prime} J} f(x)\right\|_{\mathbf{X}} & =\left\|T_{J} f(x)\right\|_{\mathbf{X}(J)}=\left\|\int_{Q} K_{J}(x-y) f(y) d y\right\|_{\mathbf{X}(J)} \\
& =\left\|\int_{Q}\left(K_{J}(x-y)-K_{J}\left(x-x_{0}\right)\right) f(y) d y\right\|_{\mathbf{X}(J)} \\
& =C\left\|\sup _{r \in J}\left|\int_{Q} \frac{1}{r^{n}}\left(\chi\left(\frac{|x-y|}{r}\right)-\chi\left(\frac{\left|x-x_{0}\right|}{r}\right)\right) f(y) d y\right|\right\|_{\mathbf{X}} \\
& \leq C\left\|\sup _{r>2 \delta} \frac{1}{r^{n}} \int_{r-\delta<|y-x|<r+\delta}|f(y)| d y\right\|_{\mathbf{X}} .
\end{aligned}
$$


The geometric argument is entirely similar to that in the proof of Theorem 2.1, although the positions of $x$ and $y$ are now interchanged. We continue now as in the proof of Theorem 2.1, that is we use Hölder's inequality with some exponent $s>1$, such that $\mathbb{X}$ is $s$-convex. We get

$$
\begin{aligned}
\left\|\mathcal{M}_{J} f(x)\right\|_{\mathbf{x}} & \leq C\left\|\left(\int_{Q} H(x-y)|f(y)|^{s} d y\right)^{1 / s}\right\|_{\mathbf{x}} \leq \quad \text { (by s-convexity) } \\
& \leq C\left(\int_{Q} H(x-y)\|f(y)\|_{\mathbf{x}}^{s} d y\right)^{1 / s} \leq c\|f\|_{L_{\mathbf{x}}^{\infty}} \text { for } \quad x \notin \tilde{Q}
\end{aligned}
$$

The restriction on $f$ to have average 0 over $Q$ can be easily removed by adding and subtracting the average. Then we can pass from $\mathcal{M}_{x, s}$ to $\mathcal{M}_{j}$ by using $|f|$ in place of $f$. Therefore, we have, for every bounded $\mathbb{X}$-valued $f$ supported in $Q$ and $x \notin \tilde{Q}$

$$
\left\|\mathcal{M}_{J} f(x)\right\|_{\mathbf{x}} \leq C\|f\|_{L_{\mathbf{x}}^{\infty}}
$$

Now if $Q^{*}$ is a cube of the same size as $\tilde{Q}$, having just one common face with $\tilde{Q}$, since $Q^{*} \subset \mathbb{R}^{n} \backslash \tilde{Q}$ almost everywhere, we see that the $B M O$ function $\left\|\mathcal{M}_{J} f(x)\right\|_{\mathrm{x}}$ has an average over $Q^{*}$ which is bounded by $C\|f\|_{L_{\mathbf{x}}^{\infty}}$. But for a $B M O$ function like $\left\|\mathcal{M}_{J} f(x)\right\|_{\mathbf{x}}$, the difference between its averages over $\tilde{Q}$ and $Q^{*}$ is bounded by its $B M O$ norm, which is itself bounded by $C\|f\|_{L_{\mathbf{x}}^{\infty}}$. Consequently, we also have

$$
\int_{\bar{Q}}\left\|\mathcal{M}_{J} f(x)\right\|_{\mathbf{x}} d x \leq C\|f\|_{L_{\mathbf{x}}^{\infty}}|Q|
$$

and the proof is finished.

It is interesting to compare the behaviour of the Hardy-Littlewood maximal function with that of the fractional maximal operator defined by

$$
\mathcal{M}_{J}^{x} f(x)=\sup _{r \in J} \frac{1}{|B(x, r)|^{1-\frac{x}{n}}} \int_{B(x, r)}|f(y)| d y, \quad 0<\alpha<n
$$

or its smooth version

$$
\mathcal{M}_{\varphi, J}^{\alpha} f(x)=\sup _{\varepsilon \in J}\left|\frac{1}{\varepsilon^{n-\alpha}} \int_{\mathbf{R}^{n}} \varphi\left(\frac{|x-y|}{\varepsilon}\right) f(y) d y\right|, \quad 0<\alpha<n .
$$

When the subindex $J$ is not necessary, in particular for Köthe function spaces, we shall simply write $\mathcal{M}^{\alpha}$ and we shall use the consistent notation $M^{\alpha}$ for the scalar version.

We have the following result.

Theorem 2.6. Let $\mathbb{X}$ be a Banach lattice which is $r$-convex, with $1<r$. Then the 
operators $\mathcal{M}_{j}^{z}$ are bounded from $L_{\mathbf{x}}^{n / x}\left(\mathbb{R}^{n}\right)$ to $B M O_{\mathbf{x}}\left(\mathbb{R}^{n}\right)$ uniformly in $J$ and if $r \geq \frac{n}{x}$, we even have the boundedness $\mathcal{M}_{J}^{x}: L_{\mathbf{X}}^{n / x}\left(\mathbb{R}^{n}\right) \rightarrow L_{\mathbf{X}}^{\infty}\left(\mathbb{R}^{n}\right)$ uniformly in $J$.

Proof. We can obviously use $\mathcal{M}_{x, J}^{\alpha}$ instead of $\mathcal{M}_{J}^{\alpha}$. Given $f$ and $Q$ we proceed as we did in Theorem 2.3 for $\mathcal{M}$. We write $f=f_{1}+f_{2}$, where $f_{1}=f \chi_{\bar{Q}}$. The difference is that now, for the "local" part $f_{1}$, we can simply control $\mathcal{M}_{J}^{\alpha} f_{1}$ or $\mathcal{M}_{x, J}^{\alpha} f_{1}$ by $I_{x}\left|f_{1}\right|$, where $I_{\alpha}$ is the fractional integral given by

$$
I_{\alpha} g(x)=\int_{\mathbf{R}^{n}} \frac{g(y) d y}{|x-y|^{n-\alpha}}
$$

But $I_{\alpha}$ is a convolution operator with a positive kernel, and consequently, the HardyLittlewood-Sobolev theorem is still valid for $\mathbb{X}$-valued functions; in other words, $I_{\alpha}$ is bounded from $L^{p}$ to $L^{q}$, provided $p<n / \alpha$ and $1 / q=1 / p-(\alpha / n)$. We use this to get

$$
\begin{aligned}
& \frac{1}{|Q|} \int_{Q}\left\|\mathcal{M}_{J}^{\alpha} f_{1}(x)\right\|_{\mathrm{x}} d x \leq\left(\frac{1}{|Q|} \int_{Q}\left\|I_{\alpha} f_{1}(x)\right\|_{\mathrm{x}}^{q} d x\right)^{1 / q} \\
& \leq \frac{C}{|Q|^{1 / q}}\left(\int_{\bar{Q}}\|f(x)\|_{\mathbf{X}}^{p} d x\right)^{1 / p} \leq \frac{C}{|Q|^{1 / q}}\left(\int_{\tilde{Q}}\|f(x)\|_{\mathbf{X}}^{p_{x p}^{n}} d x\right)^{x / n}|\tilde{Q}|^{\frac{1 / p}{\left(\frac{n}{p p}\right)}} \leq\|f\|_{L_{\mathbf{X}}^{n / x}\left(\mathbf{R}^{n}\right)}
\end{aligned}
$$

since

$$
\frac{1 / p}{\left(\frac{n}{x p}\right)^{\prime}}=\frac{1}{q}
$$

Now for $f_{2}$, we have to estimate the $\mathbb{X}$-norm of

$$
\begin{aligned}
& \sup _{\varepsilon} \frac{1}{\varepsilon^{n-\alpha}} \int_{R^{n}}\left|\chi\left(\frac{|x-y|}{\varepsilon}\right)-\chi\left(\frac{\left|x_{0}-y\right|}{\varepsilon}\right)\right|\left|f_{2}(y)\right| d y \\
& \quad \leq \sup _{e>\delta} \frac{1}{\varepsilon^{n-\alpha}} \int_{\varepsilon-\delta<\left|x_{0}-y\right|<\epsilon+\delta}|f(y)| d y \\
& \quad \leq \sup _{e>\delta} \frac{1}{\varepsilon^{n-z}}\left(\int_{\varepsilon-\delta<\left|x_{0}-y\right|<\varepsilon+\delta}|f(y)|^{r} d y\right)^{1 / r}\left\{c_{n}\left((\varepsilon+\delta)^{n}-(\varepsilon-\delta)^{n}\right)\right\}^{1 / r^{\prime}} \\
& \quad=C \sup _{e>\delta}\left(\int_{R^{n}} g_{\varepsilon}\left(x_{0}-y\right)|f(y)|^{r} d y\right)^{1 / r},
\end{aligned}
$$

where

$$
g_{e}(x)=\frac{\left\{c_{n}\left((\varepsilon+\delta)^{n}-(\varepsilon-\delta)^{n}\right)\right\}^{r / r^{\prime}}}{\varepsilon^{(n-a) r}} \chi_{e-\delta<|x|<e+\delta}(x) \leq C \frac{\delta^{r / r^{\prime}}}{|x|^{n-r a+r-1}} \chi_{|x|>\delta}=G(x)
$$

Then 


$$
\begin{aligned}
& \left\|\sup _{e>\delta}\left(\int_{\mathbf{R}^{n}} g_{e}\left(x_{0}-y\right)|f(y)|^{r} d y\right)^{1 / r}\right\|_{\mathbf{X}} \\
& \leq\left\|\left(\int_{\mathbf{R}^{n}} G\left(x_{0}-y\right)|f(y)|^{r} d y\right)^{1 / r}\right\|_{\mathbf{X}} \quad \text { (since } \mathbb{X} \text { is } r \text {-convex) } \\
& \leq\left(\int_{\mathbf{R}^{n}} G\left(x_{0}-y\right)\|f(y)\|_{\mathbf{X}}^{r} d y\right)^{1 / r}
\end{aligned}
$$

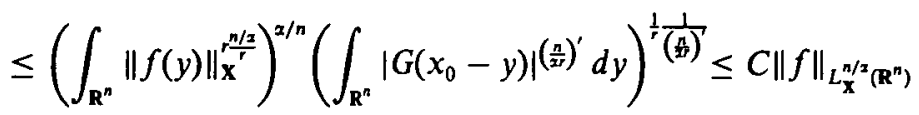

since $|G|^{\left(\frac{n}{x}\right)^{\prime}}$ is an integrable function with integral independent of $\delta$.

If $r>n / \alpha$, we simply have to observe that, by using Hölder's inequality,

$$
\mathcal{M}_{J}^{\alpha} f(x)=\sup _{r \in J}\left(\int_{B(x, r)}|f(y)|^{n / x} d y\right)^{x / n}=\left(\int_{B\left(x, r_{0}\right)}|f(y)|^{n / x} d y\right)^{\alpha / n}
$$

if $r_{0}$ denotes the maximum radius in $J$. Then we use the $n / \alpha$-convexity to get

$$
\left\|\mathcal{M}_{J}^{\alpha} f(x)\right\|_{\mathbf{x}} \leq\left(\int_{B\left(x, r_{0}\right)}\|f(y)\|_{\mathbf{X}}^{n / x} d y\right)^{x / n} \leq\|f\|_{L_{\mathbf{x}}^{n / x}\left(\mathbf{R}^{n}\right)} .
$$

Next we shall present an example to show that the convexity requirements in Theorem 2.6 are really needed.

Proposition 2.7. Let $0<\alpha<1$. Then $\mathcal{M}^{x}$ is bounded from $L_{\ell^{p}}^{1 / \alpha}([0,1])$ to $L_{\ell^{p}}^{\infty}[(0,1)]$ if and only if $p \geq \frac{1}{x}$.

Proof. Let $p<\frac{1}{a}$. We are going to construct $f \in L_{\ell^{p}}^{1 / z}([0,1])$ such that $\mathcal{M}^{\alpha} f \notin L_{\ell^{p}}^{\infty}([0,1])$. Define $f(x)=\left(f_{j}(x)\right)_{j=1}^{\infty}=\left(a_{j} \chi_{\left[2^{-j}, 2^{-j+1}\right.}\right)_{j=1}^{\infty}$ for appropriate $a_{j}>0$. We start by choosing the $a_{j}^{\prime}$ so that we have

$$
\int_{0}^{1}\|f(x)\|_{\ell^{p}}^{1 / 2}=\sum_{j=1}^{\infty} a_{j}^{1 / 2} 2^{-j}<\infty
$$

Now, if $0<x<2^{-j}, M^{\alpha}\left(f_{j}\right)(x)=\frac{a j^{-j}}{\left(2^{-j+1}-x\right)^{-s}}$. In particular, given $n \in \mathbb{N}$, if $j \leq n$ and $0<x<2^{-n}, M^{\alpha}\left(f_{j}\right)(x) \geq C a_{j} 2^{-j x}$. It follows that, for $0<x<2^{-n}$

$$
\left\|\mathcal{M}^{x}(f)(x)\right\|_{\iota^{p}}=\left(\sum_{j=1}^{\infty}\left(M^{z} f_{j}(x)\right)^{p}\right)^{1 / p} \geq C\left(\sum_{j=1}^{n}\left(a_{j} 2^{-j x}\right)^{p}\right)^{1 / p} .
$$

Now if we choose the $\alpha_{j}^{\prime} \mathrm{s}$ in such a way that the sequence $\left(a_{j} 2^{-j x}\right)$ belongs to $\ell^{1 / x}$, but 
it does not belong to $\ell^{p}$, which is possible since $\ell^{p} \subsetneq \ell^{1 / a}$, we make sure that $\mathcal{M}^{x} f \notin L_{\ell^{p}}^{\infty}[0,1]$

\section{Convexity is not sufficient for the H.L. property}

The main purpose of this section is to present an example, based upon one given by Bourgain in [3] for the U.M.D. property, which shows that the assumption that a Banach lattice $\mathbb{X}$ is $p$-convex for some $p>1$, even for large $p<\infty$, is not enough to guarantee that $\mathbb{X}$ satisfies the H.L. property. The example is based upon the following simple observation.

Proposition 3.1. Let $\mathbb{X}$ be a Banach lattice of functions on the torus $\mathbb{T}$, whose norm is translation invariant. Then if $\mathrm{X}$ satisfies the H.L. property, necessarily the HardyLittlewood maximal operator $M$ must be bounded in $\mathbb{X}$ with a norm depending only on the norm of $\mathcal{M}$ on the spaces $L_{\mathbf{x}}^{p}(\mathbb{T})$.

Proof. Fix $0<p<\infty$. Then we know that $\mathcal{M}$ is bounded in $L_{\mathbf{x}}^{p}(\mathbb{T})$. Given a function $\varphi \in \mathbb{X}$, we define $f: \mathbb{T} \rightarrow \mathbb{X}$ by making $f(x)=\varphi_{x}$, where $\varphi_{x}$ denotes the translated function $\varphi_{x}(t)=\varphi(t+x)$. Since the norm of $\mathbb{X}$ is translation-invariant, we have $\|f\|_{L_{\mathbf{x}}^{p}(\mathrm{~T})}=\|\varphi\|_{\mathbf{x}}$. On the other hand, $\mathcal{M} f(x)=(M \varphi)_{x}$, and, consequently

$$
\|M \varphi\|_{\mathbf{x}}=\left(\int_{\mathbf{T}}\left\|(M \varphi)_{\mathbf{x}}\right\|_{\mathbf{x}}^{p}\right)^{1 / p}=\left(\int_{\mathbf{T}}\|\mathcal{M} f(x)\|_{\mathbf{x}}^{p}\right)^{1 / p} \leq C\left(\int_{\mathbf{T}}\|f(x)\|_{\mathbf{X}}^{p}\right)^{1 / p}=C\|\varphi\|_{\mathbf{x}}
$$

Therefore, what we need to do is to construct a Banach lattice with translationinvariant norm which is $p$-convex for a fixed $p>1$ and such that $M$ is not bounded in $\mathbb{X}$. Actually, it is enough if we are able to build a family of lattices $\mathbb{X}_{N}$, all of them translation-invariant and $p$-convex so that the norm of $M$ in $\mathbb{X}_{N}$ tends to $\infty$ as $n \rightarrow \infty$, because then we can simply consider the $\ell^{p}$-direct sum of all the $\mathbb{X}_{N}$, and that will be a lattice still $p$-convex on which the maximal operator is unbounded.

The key to the construction is the following result.

Lemma 3.2. Let $1<p<\infty$ and $N \in \mathbb{N}$. Then there exist a function $\varphi:[0,1] \rightarrow[0, \infty[$ and a Borel set $E \subset[0,1]$, actually a finite union of intervals, such that
(a) $\int_{0}^{1} \varphi(x)^{p} d x=1$
(b) $\int_{E+t} \varphi(x)^{p} d x \leq 1 / N$ for all $0 \leq t \leq 1$ where $E+t=\{x+t$ modulo $1: x \in E\}$
(c) $\int_{E}(M \varphi)(x)^{p} d x \geq(1 / 3)^{p}$.

Proof. For every $j$ such that $1 \leq j \leq N$, we define 


$$
\begin{gathered}
x_{j}= \begin{cases}2^{-j} & \text { if } j<N \\
0 & \text { if } j=N\end{cases} \\
\delta_{j}=2^{-2 j-N+1} \quad \text { and } \quad c_{j}^{p}=\frac{1}{N \delta_{j}}=\frac{2^{2 j+N-1}}{N} .
\end{gathered}
$$

Then we consider the sets $D_{j}=\left[x_{j}, x_{j}+\delta_{j}\right]$, for all $j \leq N, E_{j}=\left[x_{j}+2 \delta_{j}, x_{j}+3 \delta_{j}\right]$ if $j<N$ and $E_{N}=D_{N}$. Finally, we let

$$
\varphi=\sum_{j=1}^{N} c_{j} \chi_{D_{j}} \quad E=\bigcup_{j=1}^{N} E_{j} \quad \text { and } \quad D=\bigcup_{j=1}^{N} D_{j}
$$

We shall see that the function $\varphi$ has the three required properties.

$$
\begin{gathered}
\int_{0}^{1} \varphi(x)^{p} d x=\sum_{j=1}^{N} c_{j}^{p}\left|D_{j}\right|=\sum_{j=1}^{N} \frac{1}{N \delta_{j}} \delta_{j}=1 . \\
\int_{E} \varphi(x)^{p} d x=c_{N}^{p}\left|D_{N}\right|=\frac{1}{N}
\end{gathered}
$$

since $E \cap D=D_{N}$.

Now we just need to realize that if we fix some $E_{j}$ and we look at all the translates $D_{k}+\delta$ for a fix $\delta$ and all $k \geq j$, only one of these translates can intersect $E_{j}$. It follows that, given $\delta$, there are only one couple $j, k$, such that $E_{j}+\delta \cap D_{k} \neq \emptyset$. Consequently

$$
\int_{E+\delta} \varphi(x)^{p} d x=\int_{E_{j}+\delta} c_{k}^{p} \chi_{D_{k}}(x) d x \leq c_{k}\left|D_{j \vee k}\right| \leq c_{j \vee k}^{p}\left|D_{j \vee k}\right|=\frac{1}{N} .
$$

Finally we realize that if $x \in E_{j}$, then $M\left(\chi_{D_{j}}\right)(x) \geq 1 / 3$. Therefore

$$
\int_{E}(M \varphi)(x)^{p} d x \geq \sum_{j=1}^{N} \int_{E_{j}} c_{j}^{p}(1 / 3)^{p}=(1 / 3)^{p}
$$

Definition 3.3. Let $1<p<\infty$ and $N \in \mathbb{N}$. Then we have the set $E=E(p, N)$ constructed as in Lemma 3.2. We use it to define a Banach lattice $\mathbb{X}_{p, N}$ of functions on $[0,1]$, by means of the function norm

$$
\|f\|_{\mathbf{x}_{p . N}}=\sup _{t \in[0,1]}\left\|f \chi_{E+t}\right\|_{p} .
$$

Then we have the following result.

Proposition 3.4. $\mathbb{X}_{p, N}$ is a Banach lattice of functions on $[0,1]$, with translation- 
invariant norm, which is $r$-convex for every $r$ such that $1 \leq r<p$, and on which the maximal operator has a norm $\geqq \frac{N^{1 / p}}{3}$.

Proof. That the norm is translation-invariant follows immediately from its definition. Also, the existence of the function $\varphi$ in Lemma 3.2, gives

$$
\|\varphi\|_{\mathbf{x}_{p, N}}^{p} \leq \frac{1}{N} \quad\|M \varphi\|_{\mathbf{x}_{p, N}}^{p} \geq\left(\frac{1}{3}\right)^{p}
$$

so that the norm of $M$ in $\mathbb{X}_{p, N}$ is $\geq \frac{N^{1 / p}}{3}$.

It only remains for us to prove that the lattice $\mathbb{X}_{p, N}$ is $r$-convex for every $1 \leq r<p$. The reason is that, in the terminology of $[12], \mathbb{X}_{p, N}$ satisfies an upper $p$-estimate with constant 1 . This means that, for every choice of functions $f_{j}$ with pairwise disjoint supports

$$
\left\|\sum_{j=1}^{n} f_{j}\right\|_{\mathbf{x}_{p, N}} \leq\left(\sum_{j=1}^{n}\left\|f_{j}\right\|_{\mathbf{x}_{p, N}^{p}}\right)^{1 / p} .
$$

This inequality is immediate from the definition of the norm. Then we simply have to use the result of Maurey and Pisier that if a Banach lattice satisfies an upper $p$ estimate, then it is $r$-convex for $1<r<p$, even though it may fail to be $p$-convex. A proof of this result can be seen in [12, Theorem 1.f.7].

\section{The image of $L L_{\mathbf{x}}^{\infty}$ in $B M O_{\mathbf{x}}$}

For any Banach space $\mathbb{X}$, the Hardy space $H_{\mathbf{X}}^{\prime}$ is defined in the usual way (as in [14]) by means of atoms. It is a well known fact, due to J. Garcia-Cuerva and Bourgain (see [5]) that

$$
H_{\mathbf{x}}^{1}=\left\{f \in L_{\mathbf{x}}^{1}: G(f) \in L_{\mathbf{x}}^{1}\right\}
$$

where $G(f)$ is the "grand" maximal function, defined by $G(f)(x)=\sup _{e>0}\left\|\varphi_{e} * f(x)\right\|$ for $\varphi$ in a nice class of smooth functions.

If $\mathbb{X}$ is U.M.D.

$$
H_{\mathbf{x}}^{1}=\left\{f \in L_{\mathbf{x}}^{1}: H(f) \in L_{\mathbf{x}}^{1}\right\}
$$

where $H$ is the Hilbert transform.

If $\mathbb{X}$ is a H.L. Banach lattice

$$
H_{\mathbf{x}}^{\prime}=\left\{f \in L_{\mathbf{x}}^{1}: \mathcal{M}_{\varphi, J}(f) \in L_{\mathbf{x}}^{\prime} \text { uniformly }\right\}
$$

If X is U.M.D., we have $\left(H_{\mathbf{x}^{*}}^{1}\right)^{*}=B M O_{\mathbf{x}}$. This duality, together with the characterization 
of $H^{1}$ by means of the Hilbert transform $H$, leads to the following description of $B M O_{\mathbf{x}}$

$$
B M O_{\mathbf{X}}=\left\{f+H g: f, g \in L_{\mathbf{X}}^{\infty}\right\}
$$

(see the first chapter of [9], where this is done in detail for the scalar case).

Now we ask ourselves if the characterization of $H_{\mathrm{X}}^{1}$ by means of $\mathcal{M}_{\varphi}$ for $\mathbb{X}$ a $H . L$. Banach lattice will have as a consequence a parallel characterization of $B M O_{\mathbf{x}}$. We shall present a very simple negative result which shows the difference in behaviour between $\mathcal{M}_{\varphi}$ and a "typical" singular integral. The singularities of $\mathcal{M}_{\varphi} f$ for $f \in L_{\mathbf{X}}^{\infty}$ are better than those of a typical function in $B M O_{\mathbf{x}}$ and they get better as the lattice gets more convex. In the limit case of $\infty$-convexity (the scalar case or $\ell^{\infty}$ ) we get, of course, bounded functions. We shall present this result for the torus $\mathbb{T}^{n}$.

Theorem 4.1. Let $\mathbb{X}$ be a Banach lattice with the H.L. property and r-convex with a given $r>1$. Then for every $f \in L_{\mathbf{X}}^{\infty}\left(\mathbb{T}^{n}\right)$ and for every cube $Q$

$$
\frac{1}{|Q|} \int_{Q}\left\|\mathcal{M}_{J} f(x)\right\|_{\mathbf{x}} d x \leq C\left(\log \frac{1}{|Q|}\right)^{1 / r}\|f\|_{L_{\mathbf{x}}^{\infty}}
$$

with $C$ independent of $f$ and $Q$.

Proof. Given $Q$ and $f$, we write $f=f_{1}+f_{2}$, where $f_{1}=f \chi_{\bar{Q}}$. Then $\mathcal{M}_{J} f(x) \leq$ $\mathcal{M}_{J} f_{1}(x)+\mathcal{M}_{J} f_{2}(x)$, and we estimate the two terms separately.

The first term gives, by using the H.L. property with some $p>1$

$$
\frac{1}{|Q|} \int_{Q}\left\|\mathcal{M}_{J} f_{1}(x)\right\|_{\mathbf{x}} d x \leq\left(\frac{1}{|Q|} \int_{Q}\left\|\mathcal{M}_{J} f_{1}(x)\right\|_{\mathbf{x}}^{p} d x\right)^{1 / p} \leq C\left(\frac{1}{|Q|} \int_{\bar{Q}}\|f(x)\|_{\mathbf{X}}^{p} d x\right)^{1 / p} \leq C\|f\|_{L_{\mathbf{x}}^{\infty}}
$$

which is even better than the estimate we are looking for, since the logarithmic term is absent.

For the second term we have

$$
\begin{aligned}
\mathcal{M}_{J} f_{2}(x) \leq \mathcal{M}_{\varphi, J}\left(\left|f_{2}\right|\right)(x) & =\sup _{\varepsilon \in J} \frac{1}{c_{0} \varepsilon^{n}} \int_{\mathbb{T}^{n}} \varphi\left(\frac{|x-y|}{\varepsilon}\right)\left|f_{2}(y)\right| d y \\
& \leq \sup _{\varepsilon \in J}\left(\frac{1}{c_{0} \varepsilon^{n}} \int_{\pi^{n}} \varphi\left(\frac{|x-y|}{\varepsilon}\right)\left|f_{2}(y)\right|^{r} d y\right)^{1 / r} \leq\left(\int_{T^{n} \backslash \grave{Q}} \frac{C}{|x-y|^{n}}|f(y)|^{r} d y\right)^{1 / r} .
\end{aligned}
$$

Since $\mathbb{X}$ is $r$-convex

$$
\begin{aligned}
\left\|\mathcal{M}_{J} f_{2}(x)\right\|_{\mathbf{X}} & \leq\left\|\left(\int_{\mathbf{T}^{n} \backslash \bar{Q}} \frac{C}{|x-y|^{n}}|f(y)|^{r} d y\right)^{1 / r}\right\|_{\mathbf{X}} \leq\left(\int_{\mathbf{T}^{n} \backslash \bar{Q}} \frac{C}{|x-y|^{n}}\|f(y)\|_{\mathbf{X}}^{r} d y\right)^{1 / r} \\
& \leq C\|f\|_{L_{\mathbf{X}}^{\infty}}\left(\int_{|Q|^{1 / n}}^{K} \frac{d t}{t}\right)^{1 / r} \leq C\|f\|_{L_{\mathbf{X}}^{\infty}}\left(\log \frac{1}{|Q|}\right)^{1 / r} .
\end{aligned}
$$


As an application, we give a sufficient condition for the boundedness from $L_{\mathbf{X}}^{\infty}$ to $B M O_{\mathrm{x}}$ of the commutator

$$
\sup _{e} \frac{1}{\varepsilon^{n}}\left|\int_{\mathbf{T}^{n}}(b(x)-b(y)) \chi\left(\frac{|x-y|}{\varepsilon}\right) f(y) d y\right|=\mathcal{M}_{b} f(x)
$$

By restricting $\varepsilon$ to a finite set $J$ of positive rationals, we have the corresponding operators $\mathcal{M}_{b, J}$.

Theorem 4.2. If $\mathbb{X}$ is a H.L. Banach lattice which is $r$-convex for some $r>1$, then a sufficient condition for the boundedness of $\mathcal{M}_{b}$ from $L_{\mathbf{X}}^{\infty}\left(\mathbb{T}^{n}\right)$ to $B M O_{\mathbf{x}}\left(\mathbb{T}^{n}\right)$ (i.e. uniform boundedness of the operators $\left.\mathcal{M}_{b, J}\right)$ is that $b \in B M O_{\left(\log _{x} \frac{1}{x}\right)}^{-1 / r}$, by which we mean that

$$
\frac{1}{|Q|} \int_{Q}\left|b(x)-b_{Q}\right| d x \leq C\left(\log \frac{1}{|Q|}\right)^{-1 / r} \text {. }
$$

The condition (4.3) defines a subspace of $B M O_{\mathbf{x}}\left(\mathbb{T}^{n}\right)$. Of course, in the limit case $r=\infty$, which includes the scalar case, we simply need $b \in B M O$.

Proof. If we write $T$ for the linearization of $\mathcal{M}$, forgetting about the subindex $J$ to simplify notation, and consider the commutator

$$
T_{b} f(x)=b(x) T f(x)-T(b f)(x),
$$

it will be enough to establish the uniform boundedness of $T_{b, J}$ from $L_{\mathbf{x}}^{\infty}$ to $B M O_{\mathrm{x}(J)}$. Given $Q$, we write, exactly as in the proof of Theorem $2.1, f=f_{1}+f_{2}$, where $f_{1}=f \chi_{\bar{Q}}$. Then, for $x, u \in Q$, we have

$$
\begin{aligned}
T_{b} f(x) & -\left(T_{b} f_{Q}=T_{b} f_{1}(x)-\left(T_{b} f_{1}\right)_{Q}+\left(b(x)-b_{Q}\right) T f_{2}(x)\right. \\
& -T\left(\left(b-b_{Q}\right) f_{2}\right)(x)-\frac{1}{|Q|} \int_{Q}\left(b(z)-b_{Q}\right) T f_{2}(z)+\frac{1}{|Q|} \int_{Q} T\left(\left(b-b_{Q}\right) f_{2}\right)(z) d z \\
& =T_{b} f_{1}(x)-\left(T_{b} f_{1}\right)_{Q}+\left(b(x)-b_{Q}\right)\left(T f_{2}(x)-T f_{2}(u)\right)+\left(b(x)-b_{Q}\right) T f_{2}(u) \\
& -\frac{1}{|Q|} \int_{Q}\left(b(z)-b_{Q}\right)\left(T f_{2}(z)-T f_{2}(u)\right) d z \\
& +\frac{1}{|Q|} \int_{Q}\left\{T\left(\left(b-b_{Q}\right) f_{2}\right)(z)-T\left(\left(b-b_{Q}\right) f_{2}\right)(x)\right\} d z \\
& =\sigma_{1}(x)-\left(\sigma_{1}\right)_{Q}+\sigma_{2}(x, u)+\sigma_{4}(x, u)-\left(\sigma_{2}(\cdot, u)\right)_{Q}+\left(\sigma_{3}(x, \cdot)\right)_{Q},
\end{aligned}
$$

where 


$$
\begin{aligned}
\sigma_{1} & =T_{b} f_{1}, \\
\sigma_{2}(x, u) & =\left(b(x)-b_{Q}\right)\left(T f_{2}(x)-T f_{2}(u)\right), \\
\sigma_{3}(x, z) & =T\left(\left(b-b_{Q}\right) f_{2}\right)(z)-T\left(\left(b-b_{Q}\right) f_{2}\right)(x) \text { and } \\
\sigma_{4}(x, u) & =\left(b(x)-b_{Q}\right) T f_{2}(u) .
\end{aligned}
$$

Now, since $b \in B M O, T_{b}$ is bounded in $L^{p}$ for $1<p<\infty$ (see [15]), and, consequently

$$
\begin{aligned}
\frac{1}{|Q|} \int_{Q}\left\|\sigma_{1}(x)\right\|_{\mathbf{X}(J)} d x & \leq\left(\frac{1}{|Q|} \int_{Q}\left\|T_{b} f_{1}(x)\right\|_{\mathbf{X}(J)}^{p} d x\right)^{1 / p} \\
& \leq\left(\frac{C}{|Q|} \int_{\bar{Q}}\|f(x)\|_{\mathbf{x}}^{p} d x\right)^{1 / p} \leq C\|f\|_{L_{\mathbf{x}}^{\infty}} .
\end{aligned}
$$

Also

$$
\begin{aligned}
\frac{1}{|Q|} \int_{Q}\left\|\sigma_{2}(x, u)\right\|_{\mathbf{X}(J)} d x & =\frac{1}{|Q|} \int_{Q}\left|b(x)-b_{Q}\right|\left\|T f_{2}(x)-T f_{2}(u)\right\|_{\mathbf{X}(J)} d x \\
& \leq C\|b\|_{B M O}\|f\|_{L_{\mathbf{X}}^{\infty},}
\end{aligned}
$$

as in the proof of Theorem 2.1, and

$$
\begin{aligned}
\left\|\sigma_{3}(x, z)\right\|_{\mathbf{X}(J)} & \leq\left\|\operatorname { s u p } _ { \varepsilon } \frac { 1 } { \varepsilon ^ { n } } \int _ { \mathbb { T } ^ { n } | \dot { Q } } | \chi ( \frac { | x - y | } { \varepsilon } ) - \chi ( \frac { | z - y | } { \varepsilon } ) | \left|b(y)-b_{Q}\|f(y) \mid\|_{\mathbf{X}}\right.\right. \\
& \leq C\|b\|_{B M O}\|f\|_{L_{\mathbf{x}}^{\infty}}
\end{aligned}
$$

as in the proof of Theorem 2.3 .

Finally, from our assumption that $b \in B M O_{\left(\log _{\frac{x}{x}}^{1}\right)^{-1 / r}}$ and from Theorem 4.1, we get

$$
\begin{aligned}
& \frac{1}{|Q|} \int_{Q}\left\|\sigma_{4}(x, u)\right\|_{\mathbf{X}(J)} d x \\
& \quad \leq \frac{1}{|Q|} \int_{Q}\left|b(x)-b_{Q}\right| d x\left\|\sup _{\varepsilon} \int_{\mathbf{T}^{n} \mid \bar{Q}} \frac{1}{\varepsilon^{n}} \chi\left(\frac{|u-y|}{\varepsilon}\right)|f(y)| d y\right\|_{\mathbf{X}} \\
& \quad \leq C\left(\log \frac{1}{|Q|}\right)^{-1 / r}\left(\log \frac{1}{|Q|}\right)^{1 / r}\|f\|_{L_{\mathbf{x}}^{\infty}}
\end{aligned}
$$

This finishes the proof of the theorem.

\section{REFERENCES}

1. A. Benedek, A. P. Calderón and R. Panzone, Convolution operators on Banach space valued functions, Proc. Nat. Acad. Sci. U.S.A. 48 (1962), 356-365.

2. C. Bennett, R. A. DeVore and R. Sharpley, Weak- $L^{\infty}$ and BMO, Ann. of Math. 113 (1981), 601-611. 
3. J. Bourgain, Some remarks on Banach in which martingale different sequences are unconditional, Ark. Mat. 21 (1983), 163-168.

4. J. Bourgain, Extension of a result of Benedek, Calderón and Panzone, Ark. Mat. 22 (1984), 91-95.

5. J. Bourgain, Vector-valued singular integrals and the $H^{1}-B M O$ duality, in Probability theory and Harmonic Analysis (J. A. Chao and W. A. Woyczynski (editors), M. Dekker, New York and Basel, 1986), 1-19.

6. D. L. Burkholder, A geometric condition that implies the existence of certain singular integrals of Banach space valued functions, in Proc. Conf. in Honor of Antoni Zygmund (W. Beckner, A. P. Calderón, R. Fefferman and P. W. Jones (editors), Wadsworth, NY, 1981, vol. 1), 270-286.

7. C. Fefferman and E. M. Stein, Some maximal inequalities, Amer. J. Math. 93 (1971), 107-115.

8. J. Garcia-Cuerva, R. A. Macías and J. L. Torrea, The Hardy-Littlewood property of Banach lattices, Israel J. Math. 83 (1993), 177-201.

9. J. Garcia-Cuerva and José-Luis Rubio de Francia, Weighted norm inequalities and related topics, North Holland Math. Stud. 114, 1985.

10. E. Harboure, R. A. Macias, C. Segovia and J. L. Torrea, Some estimates for maximal functions on Köthe function spaces, Israel J. Math. 90 (1995), 349-371.

11. E. Harboure, C. Segovia and J. L. Torrea, Boundedness of commutators of fractional and singular integrals for the extreme values of p, Ill. J. Math. 41 (1997), 676-700.

12. J. Lindenstrauss and L. TZAFriRI, Classical Banach spaces II. Function spaces (Springer-Verlag, Berlin, 1979).

13. J. L. Rubio DE Francia, Martingale and integral transforms of Banach space valued functions, in Probability and Banach spaces (J. Bastero and M. San Miguel (editors), Springer Lecture Notes in Mathematics 1221, 1985), 195-222.

14. J. L. Rubio de Francia, F. J. Ruiz and J. L. Torrea, Calderón-Zygmund theory for vector-valued functions, $A d v$. in math. 62 (1986), 7-48.

15. C. SEgovia and J. L. Torrea, Weighted inequalities for commutators of fractional and singular integrals, Publ. Mat. 35 (1991), 209-235.

16. A. Uchiyama and J. M. Wilson, Approximate identities and $H^{\prime}(\mathbb{R})$, Proc. Amer. Math. Soc. 88 (1983), 53-58.

García-Cuerva and TORREa

Departamento de Matemáticas, C-XV

UNIVERSIDAD AUTÓNOMA

28049, MADRID

SPAIN

E-mail addresses: jose.garcia-cuerva@uam.es joseluis.torrea@uam.es
Macías

I.N.T.E.C.

GÜEMES 3450

3000 Santa Fe

ARGENTINA

E-mail address: macias@intec.unl.edu.ar 